\title{
Heartbeat: markers of adverse outcomes in adults with severe aortic stenosis
}

Optimal management of adults with severe aortic stenosis (AS) remains controversial with several ongoing clinical trials evaluating the potential benefit of aortic valve replacement (AVR) earlier in the disease course when patients are still asymptomatic. To a large extent, these trials are based on evidence from the Contemporary Outcomes after Surgery and Medical Treatment in Patients with Severe Aortic Stenosis (CURRENT AS) registry. ${ }^{1}$ A comprehensive review article by the CURRENT-AS investigators in this issue of Heart summarises the data from this large Japanese multi-centre retrospective registry of consecutive adults with severe AS (figure 1). Both clinicians caring for patients with AS and researchers engaged in (or thinking about) clinical trials will want to read this data summary and review.

As we learn more about the disease course in adults with AS, the complex interaction between progressive valve obstruction and concurrent ventricular and vascular changes is increasingly evident. In a study of 1383 patients with severe AS undergoing AVR, Thaden and colleagues ${ }^{2}$ found that Doppler evidence of an elevated left ventricular (LV) filling pressure was present in $43 \%$ before AVR and was an independent predictor of increased mortality after AVR, even with adjustment for age, sex and other factors (HR $1.45,95 \%$ CI 1.16 to $1.81, p=0.005$ ) (figure 2).

Dr Flynn's accompanying editorial ${ }^{3}$ points out that elevated LV filling pressures in patients with severe AS are most likely related to myocardial fibrosis, which may or may not reverse after AVR. He concludes: 'The present study identifies a useful variable that will help inform our decision-making, but perhaps the greater impact of the study is that it highlights the ongoing need for increased understanding of the enthralling complexity that is aortic stenosis.'

Cardiac imaging now is a standard approach for monitoring cardiac function in patients receiving cancer therapies associated with LV dysfunction. However, measurement variability can lead to

Correspondence to Professor Catherine M Otto, Division of Cardiology, University of Washington, Seattle, WA 98195, USA; cmotto@uw.edu
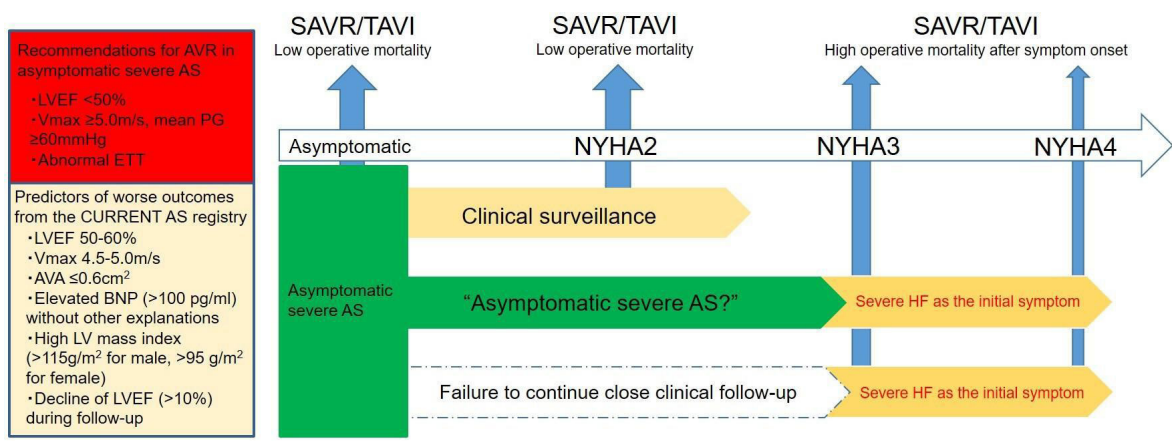

Figure 1 Potential reasons for the poor prognosis of asymptomatic patients with severe AS without aortic valve intervention. AVR is indicated in asymptomatic patients with depressed LV function ( $\mathrm{LVEF}<50 \%$ ) not due to other causes or very severe AS (Vmax $\geq 5.0 \mathrm{~m} / \mathrm{s}$ or mean aortic $P G \geq 60 \mathrm{~mm} \mathrm{Hg}$ ) and in patients who develop symptoms during exercise testing. Asymptomatic patients with severe AS should be followed up under active clinical surveillance and should be referred for SAVR or TAVI if some symptoms emerge. However, assessment of symptoms is often difficult in clinical practice, especially in the elderly, leading to false diagnosis of 'asymptomatic $\mathrm{AS}^{\prime}$. Furthermore, patients may not always be compliant to close clinical follow-up. Severe HF is often the initial symptom in a sizeable proportion under 'clinical surveillance', for whom AVR is less frequently performed than in patients without severe $\mathrm{HF}$, and mortality would be high if AVR is not performed. Operative mortality of SAVR after symptom onset is higher than that in asymptomatic patients. Furthermore, sudden death occurs not infrequently without any preceding symptom in asymptomatic patients with severe AS (1.4\%/year). Finally, AVR is anyway inevitable in a short period of time. AS, aortic stenosis; AVA, aortic valve area; AVR, aortic valve replacement; BNP, B-type natriuretic peptide; CURRENT AS, Contemporary Outcomes after Surgery and Medical Treatment in Patients with Severe Aortic Stenosis; ETT, exercise treadmill test; HF, heart failure; LV, left ventricle; LVEF, left ventricle ejection fraction; NYHA, New York Heart Association; PG, pressure gradient; SAVR, surgical aortic valve replacement; TAVI, transcatheter aortic valve replacement; Vmax, peak aortic jet velocity.

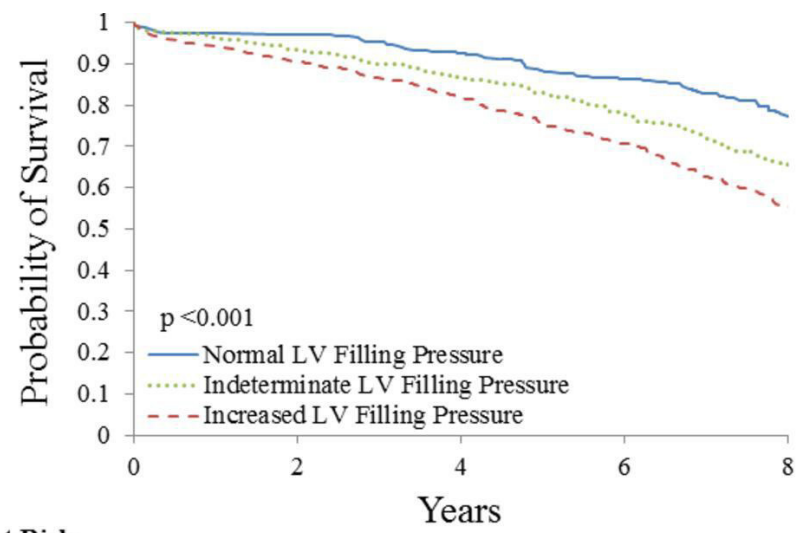

$\begin{array}{llllll}\text { At Risk: } & & & & & \\ \text { Normal } & 325 & 315 & 272 & 227 & 169 \\ \text { Indeterm } & 463 & 433 & 360 & 290 & 204 \\ \text { Increased } & 595 & 538 & 447 & 343 & 215\end{array}$

Figure 2 Long-term mortality according to echocardiographically derived LV filling pressure. There was a progressive decrease in survival in those with normal, indeterminate and increased LV filling pressure $(p<0.001)$. LV, left ventricular. 


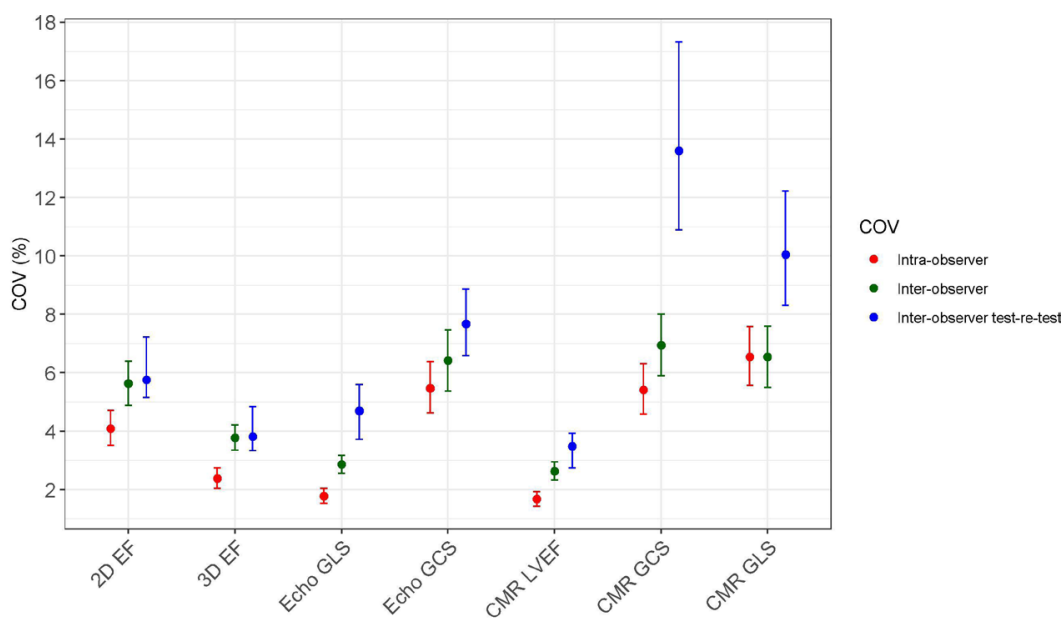

\begin{tabular}{|c|c|c|c|c|c|c|c|}
\hline & $2 \mathrm{D} \mathrm{EF}$ & $3 \mathrm{D} \mathrm{EF}$ & Echo GLS & Echo GCS & CMR LVEF & CMR GCS & CMR GLS \\
\hline Intra & $4.1[3.5,4.7]$ & $2.4[2.0,2.7]$ & $1.8[1.5,2.0]$ & $5.5[4.6,6.4]$ & $1.7[1.4,1.9]$ & $5.4[4.6,6.3]$ & $6.5[5.6,7.6]$ \\
\hline Inter & $5.6[4.9,6.4]$ & $3.8[3.3,4.2]$ & $2.9[2.6,3.2]$ & $6.4[5.4,7.5]$ & $2.6[2.3,2.9]$ & $7.0[5.9,8.0]$ & $6.5[5.5,7.6]$ \\
\hline Inter test-re-test & $5.8[5.2,7.2]$ & $3.8[3.3,4.8]$ & $4.7[3.7,5.6]$ & $7.7[6.6,8.9]$ & $3.5[2.7,3.9]$ & $13.6[10.9,17.3]$ & $10.0[8.3,12.2]$ \\
\hline
\end{tabular}

Figure 3 Interobserver, intraobserver and interobserver test-retest variability for LVEF and strain measurements with echocardiography and CMR represented as coefficient of variance (COV) and 95\% Cl. 2D, two-dimensional; 3D, three-dimensional; CMR, cardiovascular magnetic resonance; $E F$, ejection fraction; GCS, global circumferential strain; GLS, global longitudinal strain; LVEF, left ventricular ejection fraction.

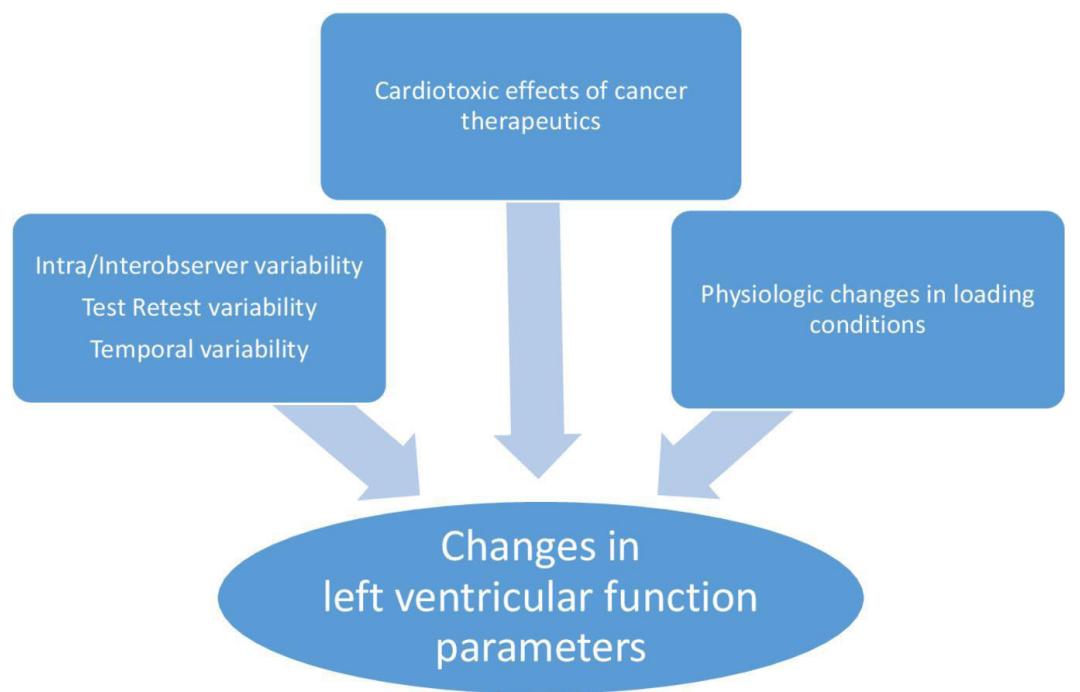

Figure 4 Factors leading to changes in left ventricular function parameters.

uncertainty in clinical decision making. In this issue of Heart, Lambert and colleagues ${ }^{4}$ report a carefully done study of temporal changes in several imaging measure of LV systolic function in 30 women receiving anthracyclines and trastuzumab for breast cancer compared with 30 control subjects. There was considerable temporal variability in all measure of LV function in both patients and control subjects. Interobsever and intraobserver variability were lowest for 2D-echocardiographic global longitudinal strain reliability with any imaging modality and especially with cardiovascular imaging in patients with cancer where small changes can lead to life-changing treatment decisions. When a decline in LVEF is observed, we must be able to distinguish between a true change in a patient's cardiac function vs an expected variability of an imperfect test' (figure 4).

In a study addressing the impact of comorbidities on outcomes in adults with ischaemic heart disease, Crowe and colleagues $^{6}$ use latent class analysis to identified five distinct comorbidity clusters. Interesting the two groups of patients at highest risk were those with vascular and musculoskeletal conditions (HR 2.38, $95 \%$ CI 2.28 to 2.49 ) and those with respiratory and musculoskeletal conditions (HR 2.62, 95\% CI 2.45 to 2.79). In the linked editorial, Bridgman ${ }^{7}$ concludes that 'The hitherto hidden mortality impact of a musculoskeletal condition when associated with either further cardiovascular disease or respiratory disease that has emerged in this study is significant. Cardiologists should be alert to these combinations in their patients and factor the increased mortality risk into their decision-making. Meeting the comorbid patient should not be a heart sink moment.'

The Education in Heart article in this issue compares the effects of lifestyle changes and pharmacological treatment on cardiovascular disease risk factors. ${ }^{8}$ As the author notes: 'Adequately implemented, changes in lifestyle achieve reductions in cardiovascular risk factors close to those of single drug therapies and should be systematically proposed to all subjects with cardiovascular risk factors.'

Funding The authors have not declared a specific grant for this research from any funding agency in the public, commercial or not-for-profit sectors.

Competing interests None declared.

Patient and public involvement Patients and/ or the public were not involved in the design, or conduct, or reporting, or dissemination plans of this research.

Patient consent for publication Not required.

Provenance and peer review Commissioned; internally peer reviewed.

(C) Author(s) (or their employer(s)) 2020. No commercia re-use. See rights and permissions. Published by BMJ. (figure 3)

In an editorial, Palaskas and LopexMattei $^{5}$ discuss the typical definition of cancer-therapeutic-related cardiac dysfunction (CRTCD) - a decline in LV ejection fraction by $10 \%$ to a value below $53 \%-55 \%$ in asymptomatic patients. The goal of periodic imaging is to detect early dysfunction and modify cancer therapy accordingly. However, 'This brings up the important issue of reproducibility and 


\section{REFERENCES}

1 Taniguchi T, Morimoto T, Takeji Y, et al. Contemporary issues in severe aortic stenosis: review of current and future strategies from the contemporary outcomes after surgery and medical treatment in patients with severe aortic stenosis registry. Heart 2020;106:802-9.

2 Thaden JJ, Balakrishnan M, Sanchez J, et al. Left ventricular filling pressure and survival following aortic valve replacement for severe aortic stenosis. Heart 2020;106:824-31.

3 Flynn AW. The enthralling complexity of aortic stenosis. Heart 2020;106:788-9.

4 Lambert J, Lamacie M, Thampinathan B, et al. Variability in echocardiography and MRI for detection of cancer therapy cardiotoxicity. Heart 2020;106:817-23.

5 Palaskas N, Lopez-Mattei J. Cancer therapy cardiotoxicity detection: understanding the limitations of cardiac imaging. Heart 2020;106:791-2.
6 Crowe F, Zemedikun DT, Okoth K, et al. Comorbidity phenotypes and risk of mortality in patients with ischaemic heart disease in the UK. Heart 2020;106:810-6.

7 Bridgman PG. The comorbid patient: does your heart sink? Heart 2020;106:790.

8 Marques-Vidal P. Comparison of lifestyle changes and pharmacological treatment on cardiovascular risk factors. Heart 2020;106:852-62. 